\title{
True ternary fission and quasifission of superheavy nuclear systems
}

\author{
A.V. Karpov ${ }^{1, a}$, V.I. Zagrebaev ${ }^{1}$, and Walter Greiner ${ }^{2}$ \\ 1 Flerov Laboratory of Nuclear Reactions, JINR, Dubna, 141980 Russia \\ 2 Frankfurt Institute for Advanced Studies, J. W. Goethe University, Frankfurt am Main, 60438 Germany
}

\begin{abstract}
We found that a true ternary fission with formation of a heavy third fragment (a new kind of radioactivity) is quite possible for superheavy nuclei due to the strong shell effects leading to a three-body clusterization with the two doubly magic tin-like cores. The three-body quasifission process could be even more pronounced for giant nuclear systems formed in collisions of heavy actinide nuclei. In this case a three-body clusterization might be proved experimentally by detection of two coincident lead-like fragments in low-energy $\mathrm{U}+\mathrm{U}$ collisions.
\end{abstract}

\section{Introduction}

Ternary fission process has already tens years history of experimental and theoretical study. The term "ternary fission" is commonly used to denote the process of formation of light charged particle accompanying fission [1]. This is a rare process (less than $1 \%$ ) relative to binary fission, see Fig. 1. The probability of such a process decreases sharply with increasing mass number of the accompanying third particle. These light particles are emitted almost perpendicularly with respect to the fission axis (equatorial emission) [1]. It is interpreted as an indication that the light ternary particles are emitted from the neck region and are accelerated by the Coulomb fields of both heavy fragments.

In contrast to such a process, the term "true ternary fission" is used for a simultaneous decay of heavy nucleus into three fragments of not very different mass [1]. Such decays of low excited heavy nuclei were not observed yet, but being discovered it becomes a new type of radioactivity. Early theoretical considerations based on the liquiddrop model (LDM) [3,4] and a more sophisticated threecenter shell model $[5,6]$ showed that the potential energy landscape and, in particular, the fission barrier plays a decisive role for the ternary fission process. It was also found that the shell effects may significantly reduce the ternary fission barriers even for much less probable oblate (triangle) deformations of very heavy nuclei. For superheavy nuclei, where the LDM fission barriers are rather low (or vanish completely), the shell correction to the total deformation energy plays even more important role. Dynamical aspects of the true ternary fission of very heavy nuclear systems (treated as a neck instability within the LDM) and its dependence on nuclear viscosity were discussed in [7]. To our knowledge, since then there was no any significant progress in theoretical (or experimental) study of ternary fission. Today it becomes possible to study experimentally

\footnotetext{
a e-mail: karpov@jinr.ru
}

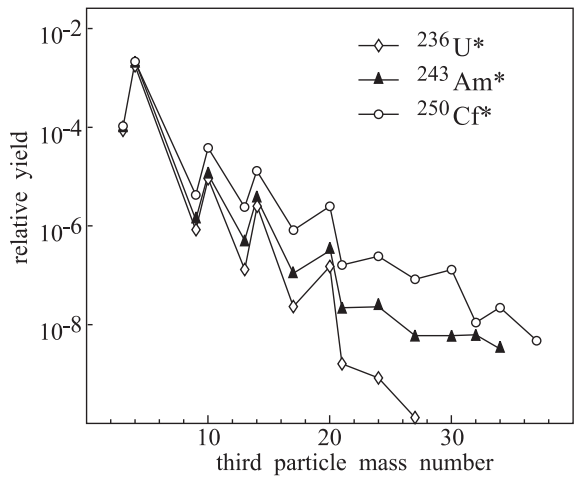

Fig. 1. Relative to binary fission yields of ternary particles in the $\left(n_{\text {th }}, f\right)$ reactions. The figure is a simplified version of Fig. 4 from [2] kindly prepared by F. Gönnenwein.

the properties and dynamics of formation and decay of superheavy nuclei [8], for which the ternary fission could be rather probable (see below).

\section{Clusterization and shape isomeric states of heavy nuclei}

The two-center shell model (TCSM) [9] looks most appropriate for calculation of the adiabatic potential energy of heavy nucleus at large dynamic deformations up to the configuration of two separated fragments. The nuclear shape in this model is determined by 5 parameters: the system elongation $R$, which for separated nuclei is the distance between their mass centers; the deformations of the two parts of the system $\delta_{1}$ and $\delta_{2}$; the mass-asymmetry $\eta=$ $\left(A_{2}-A_{1}\right) /\left(A_{2}+A_{1}\right)$, where $A_{1}$ and $A_{2}$ are the mass numbers of the system halves; and the neck parameter $\epsilon$ which smoothes the shape of overlapping nuclei.

Within the macro-microscopic approaches the energy of the deformed nucleus is composed as the sum of two 


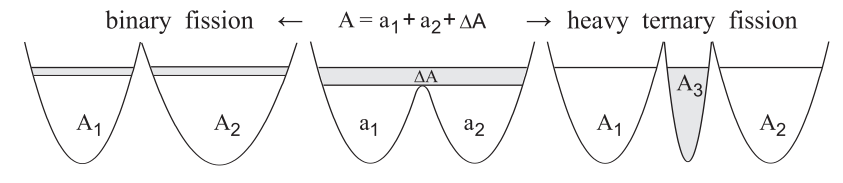

Fig. 2. Schematic view of binary and ternary fission.

parts: The macroscopic part, $E_{\mathrm{mac}}$, smoothly depends on the proton and neutron numbers and may be calculated within the LDM. The microscopic part, $\delta E$, describes the shell effects. It is constructed from the single-particle energy spectra by the Strutinsky procedure [10]. The details of calculation of the single-particle energy spectra within the TCSM, the explanation of all the parameters used as well as the extended and empirical versions of the TCSM may be found in [11].

Within the TCSM for a given nuclear configuration $\left(R, \eta, \delta_{1}, \delta_{2}\right)$ we may unambiguously determine the two deformed cores $a_{1}$ and $a_{2}$ surrounded with a certain number of shared nucleons $\Delta A=A_{\mathrm{CN}}-a_{1}-a_{2}$ (see Fig. 2). During binary fission these valence nucleons gradually spread between the two cores with formation of two final fragments $A_{1}$ and $A_{2}$. Thus, the processes of compound nucleus $(\mathrm{CN})$ formation, binary fission and quasifission may be described both in the space of the shape parameters $\left(R, \eta, \delta_{1}, \delta_{2}\right)$ and in the space $\left(a_{1}, \delta_{1}, a_{2}, \delta_{2}\right)$. This double choice of equivalent sets of coordinates is extremely important for a clear understanding and interpretation of the physical meaning of the intermediate local minima appearing on the multi-dimensional potential energy surface and could be used for extension of the model for description of three-core configurations appearing in ternary fission.

The adiabatic driving potential for formation and decay of the superheavy nucleus ${ }^{296} 116$ at fixed deformations of both fragments is shown in Fig. 3 as a function of elongation and mass asymmetry and also as a function of charge numbers $z_{1}$ and $z_{2}$ of the two cores (minimized over neutron numbers $n_{1}$ and $n_{2}$ ) at $R \leq R_{\text {cont }}$. Following the fission path (dotted curves in Fig. 3a,b) the nuclear system passes through the optimal configurations (with minimal potential energy) and overcomes the multi-humped fission barrier. The intermediate minima located along this path correspond to the shape isomeric states. These isomeric states are nothing else but the two-cluster configurations with magic or semi-magic cores surrounded with a certain amount of shared nucleons. In the case of binary fission of nucleus ${ }^{296} 116$ the second (after ground state) minimum on the fission path arises from the two-cluster nuclear configuration consisting of tin-like $\left(z_{1}=50\right)$ and krypton-like $\left(z_{2}=36\right)$ cores and about 70 shared nucleons. The third minimum corresponds to the mass-symmetric clusterization with two magic tin cores surrounded with about 30 common nucleons.

A three-body clusterization might appear just on the path from the saddle point to scission, where the shared nucleons may form a third fragment located between the two heavy clusters $a_{1}$ and $a_{2}$. In Fig. 2 a schematic view is shown for binary and ternary fission starting from the configuration of the last shape isomeric minimum of CN con-

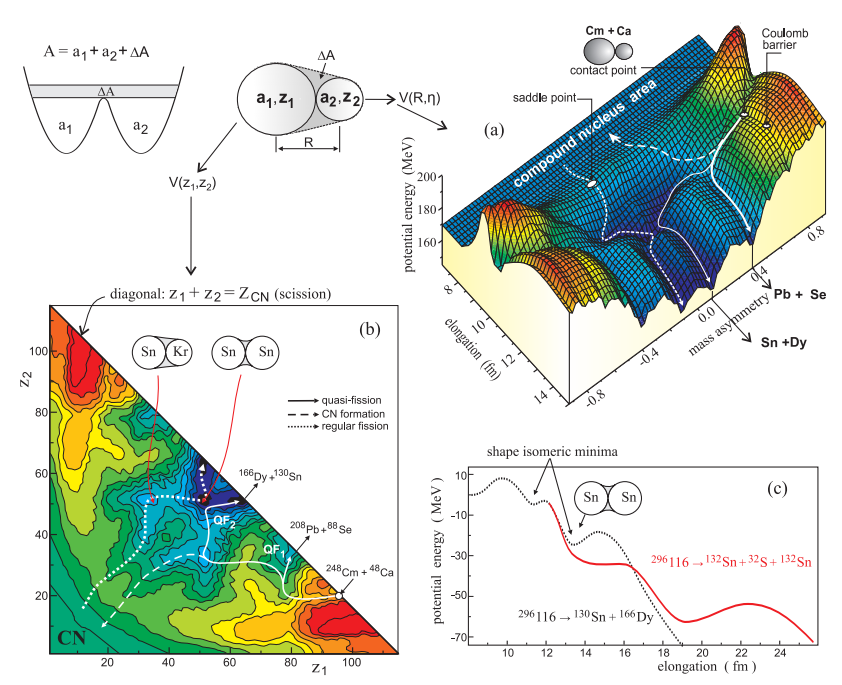

Fig. 3. Adiabatic potential energy for nucleus ${ }^{296} 116$ formed in collision of ${ }^{48} \mathrm{Ca}$ with ${ }^{248} \mathrm{Cm}$. (a) Potential energy in the "elongation-mass asymmetry" space. (b) Topographical landscape of the same potential in the $\left(z_{1}, z_{2}\right)$ plane. Dashed, solid and dotted curves show most probable trajectories of fusion, quasifission and regular fission, respectively. The diagonal corresponds to the contact configurations $\left(R=R_{\text {cont }}, z_{1}+z_{2}=Z_{\mathrm{CN}}, \Delta A=0\right)$. (c) Potential energy calculated for binary (dotted curve) and symmetric ternary fission of nucleus ${ }^{296} 116$ (see below).

sisting of two magic tin cores and about 30 extra (valence) nucleons shared between the two clusters and moving initially in the whole volume of the mononucleus. In the case of two-body fission of ${ }^{296} 116$ nucleus these extra nucleons gradually pass into one of the fragments with formation of two nuclei in the exit channel (Sn and Dy in our case, see the fission path in Fig. 3). However there is a chance for these extra nucleons $\Delta A$ to concentrate in the neck region between the two cores and form finally the third fission fragment.

\section{True ternary fission of superheavy nuclei}

There are too many collective degrees of freedom needed for proper description of the potential energy of a nuclear configuration consisting of three deformed heavy fragments. We restricted ourselves by consideration of the potential energy of a three-body symmetric configuration with two equal cores $a_{1}=a_{2}$ (and, thus, with two equal fragments $A_{1}=A_{2}$ in the exit fission channels). Also we assume equal dynamic deformations of all the fragments, $\delta_{1}=$ $\delta_{2}=\delta_{3}=\delta$, and use the same shape parametrization for axially symmetric ternary fission as in Ref. [12] (determined by three smoothed oscillator potentials).

The third fragment, $a_{3}$, appears between the two cores when the total elongation of the system, described by the variable $R$ (distance between $a_{1}$ and $a_{2}$ ), is sufficiently large to contain all three fragments, i.e., $R \geq R\left(a_{1}\right)+2 R\left(a_{3}\right)+$ $R\left(a_{2}\right)$. Finally, we calculated the three-dimensional potential energy $V\left(R, \delta, A_{3}\right)$ trying to find a preferable path for 
FUSION11
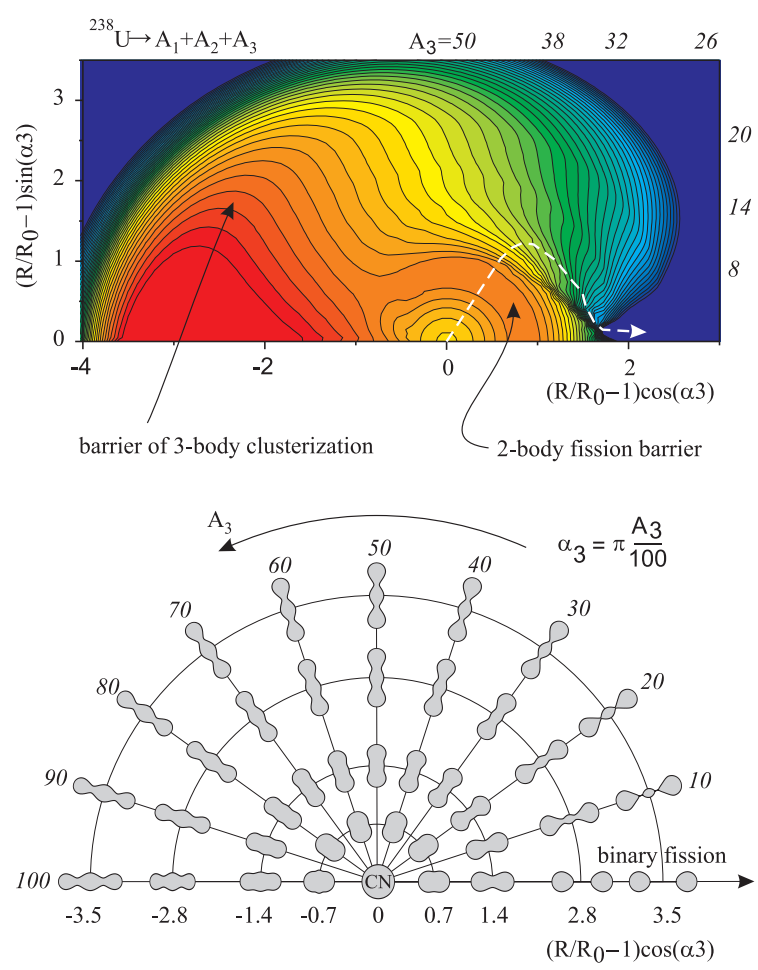

Fig. 4. Macroscopic part of the potential energy for fission of ${ }^{238} \mathrm{U}$ (upper panel) and nuclear shapes (lower panel) depending on elongation and mass of third fragment (italic numbers). The contour lines are drawn over $1 \mathrm{MeV}$. The dashed curve shows one of possible fission paths.

ternary fission and estimate how much larger the barrier is for three-body decay as compared to binary fission. For better visualization we plot the calculated potential energy $V\left(R, \delta, A_{3}\right)$ as a function of $\left(R / R_{0}-1\right) \cos \left(\alpha_{3}\right)$ and $\left(R / R_{0}-\right.$ 1) $\sin \left(\alpha_{3}\right)$ at fixed dynamic deformation $\delta=0.2$, where $\alpha_{3}=\pi \cdot A_{3} / 100$ and $R_{0}$ is the radius of sphere of equivalent volume $(\mathrm{CN})$.

The macroscopic (LDM) part of the potential energy for ${ }^{238} \mathrm{U}$ is shown in Fig. 4 along with the corresponding nuclear shapes. One may see that the potential energy has two barriers. The first one, which is closer to the ground state, is the usual barrier of binary fission. The second one is the barrier of three-body clusterization, that prevents the system from the ternary fission. Both barriers become lower as the system getting heavier. However, in the case of actinides the binary fission dominates, because after the passing over the binary fission barrier the potential energy is much steeper just in the binary exit channel (right bottom corner, $A_{3} \sim 0$ ). Emission of light third particle is possible here but not the true ternary fission. The shell correction (which makes deeper the ground state of this nucleus) does not change distinctively the total potential energy. The reason for that is quite simple. For nuclei with $Z<100$ there is just not enough charge and mass to form two doubly magic tin-like nuclei plus a third heavy fragment. Nevertheless the experiments aimed on the observation of real ternary fission of actinide nuclei (with formation of heavy third fragment) are currently in progress [13].
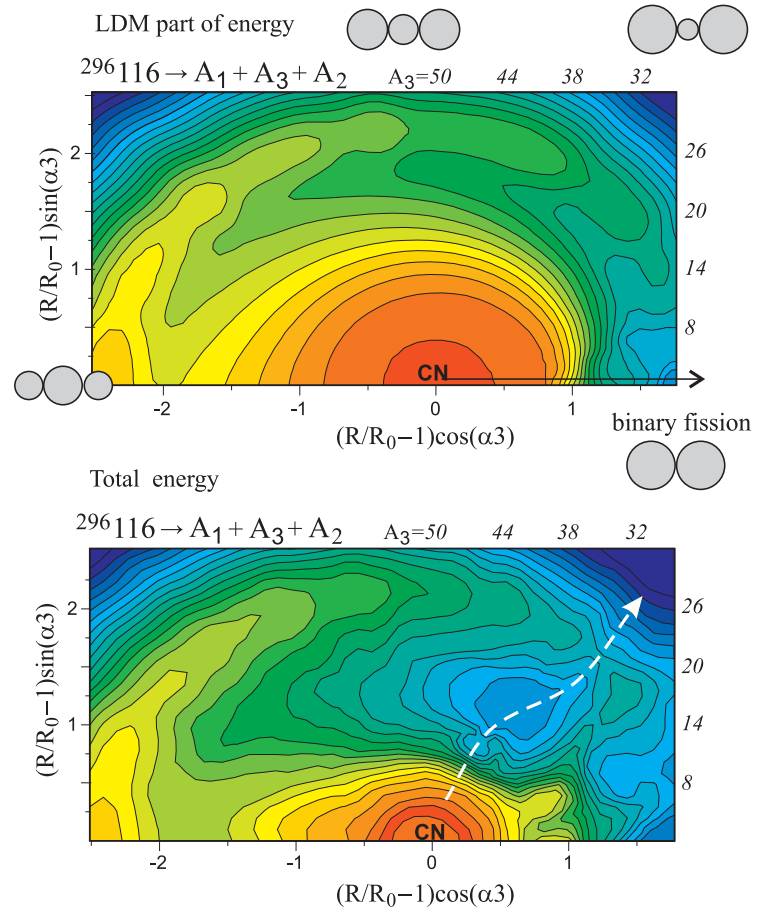

Fig. 5. Potential energy for ternary fission of superheavy nucleus ${ }^{296} 116$. Macroscopic part of potential energy and the total one (LDM plus shell corrections) are shown at the upper and lower panels, respectively, depending on elongation and mass of third fragment (italic numbers). The dashed curve shows the most probable ternary fission of nucleus ${ }^{296} 116$ onto ${ }^{132} \mathrm{Sn}+{ }^{32} \mathrm{~S}+{ }^{132} \mathrm{Sn}$.

In the case of superheavy nuclei the macroscopic potential energy does not lead to any barrier at all (neither in binary nor in ternary exit channel) and stability of these nuclei is determined completely by the shell corrections. In bottom panels of Fig. 5 the calculated potential energy is shown for superheavy nucleus ${ }^{296} 116$. In contrast with ${ }^{238} \mathrm{U}$, in this case a real possibility for ternary fission appears with formation of third fragment $A_{3} \sim 30$ and two heavy fragments $A_{1}=A_{2} \sim 130$. The ternary fission valley is quite well separated by the potential ridge from the binary fission valley. This means that the ternary fission of ${ }^{296} 116$ nucleus into the "tin-sulfur-tin" combination should dominate as compared with other true ternary fission channels of this nucleus. Even more pronounced effect may be expected for the system ${ }^{64} \mathrm{Ni}+{ }^{238} \mathrm{U}$, where the process of the ternary fission (or quasifission) will lead to formation of three magic clusters ${ }^{130} \mathrm{Sn}+{ }^{42} \mathrm{Ca}+{ }^{130} \mathrm{Sn}$.

More sophisticated consideration of the multi-dimensional potential energy surface is needed to estimate the "ternary fission barrier" accurately. However, as can be seen from Fig. 5, the height of the ternary fission barrier is not immensely high. It is quite comparable with the regular fission barrier because the ternary fission starts in fact from the configuration of the shape isomeric state which is located outside from the first (highest) saddle point of superheavy nucleus ${ }^{296} 116$ [see the solid curve in Fig. 3(c)]. 

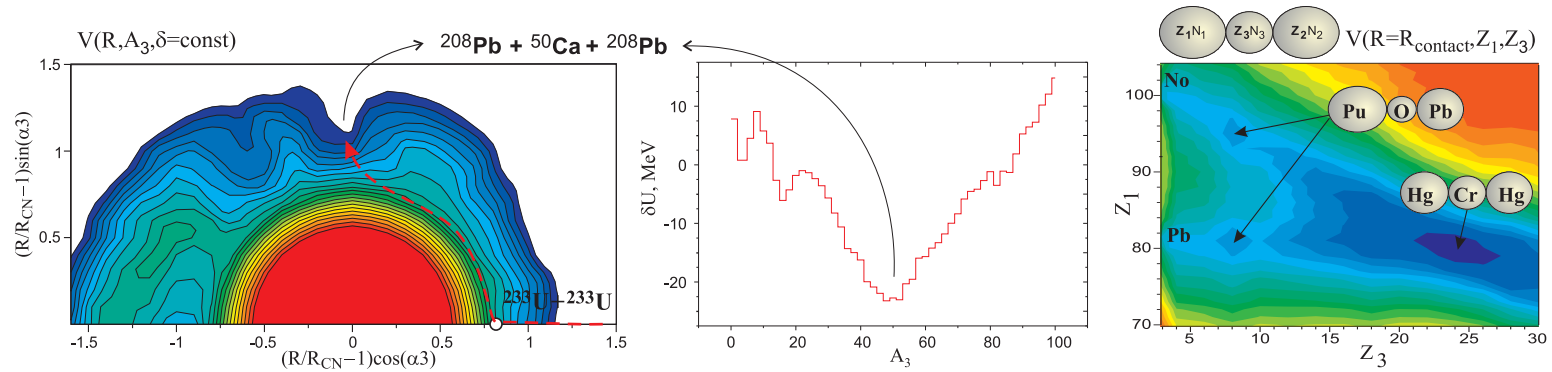

Fig. 6. (Left panel) The same as in Fig. 5 but for ${ }^{233} U+{ }^{233} U$ collision. (Middle panel) The shell correction of this system at contact configuration at zero deformation as a function of the third fragment mass. (Right panel) Landscape of potential energy of three-body contact configurations of giant nuclear system formed in collision of ${ }^{238} \mathrm{U}+{ }^{238} \mathrm{U}$.

\section{Ternary quasifission of giant nuclear systems}

Similar process of decay onto three doubly magic heavy fragments might occur also for giant nuclear systems formed in low-energy collisions of actinide nuclei. In this case compound nucleus hardly may be formed, and such decay is, in fact, a quasifission process. Conditions for the three-body decay are even better here, because the shell effects significantly reduce the potential energy of the threecluster configurations with two strongly bound lead-like fragments. In Fig. 6 the landscape of the potential energy surface is shown for a three-body clusterization of the nuclear system formed in collision of $\mathrm{U}+\mathrm{U}$. It is seen (middle panel) that the shell correction at contact configurations has a very deep minimum for the "lead-calcium-lead" $\left(A_{3}=50\right)$ clusterization.

In the right panel the potential energy is shown as a function of three variables, $Z_{1}, Z_{3}$ and $R$ (minimized over the neutron numbers) at fixed (equal) deformations of the fragments being in contact $\left(R_{1}+2 R_{3}+R_{2}=R\right)$. As can be seen, the giant nuclear system, consisting of two touching uranium nuclei, may split into the two-body exit channel with formation of lead-like fragment and complementary superheavy nucleus (the so-called anti-symmetrizing quasifission process which may lead to an enhanced yield of SH nuclei in multi-nucleon transfer reactions [14]). Beside the two-body $\mathrm{Pb}-\mathrm{No}$ clusterization and the shallow local three-body minimum with formation of light intermediate oxygen-like cluster, the potential energy has the very deep minimum corresponding to the $\mathrm{Pb}-\mathrm{Ca}-\mathrm{Pb}-$ like configuration (or $\mathrm{Hg}-\mathrm{Cr}-\mathrm{Hg}$ ) caused by the $N=126$ and $Z=82$ nuclear shells. In the right panel of Fig. 6 the potential energy of the giant nuclear system formed in collision of ${ }^{233} \mathrm{U}+{ }^{233} \mathrm{U}$ is shown as a function of $\left(R / R_{0}-1\right) \cos \left(\alpha_{3}\right)$ and $\left(R / R_{0}-1\right) \sin \left(\alpha_{3}\right)$ (see above). A possible ternary decay of this system into ${ }^{208} \mathrm{~Pb}+{ }^{50} \mathrm{Ca}+{ }^{208} \mathrm{~Pb}$ is shown by the dashed curve.

\section{Summary}

Thus we found that for superheavy nuclei the three-body clusterization (and, hence, real ternary fission with a heavy third fragment) is quite possible. The simplest way to discover this phenomenon is a detection of two tin or xenonlike clusters in low-energy collisions of medium mass nuclei with actinide targets, e.g., in ${ }^{64} \mathrm{Ni}+{ }^{238} \mathrm{U}$ reaction. These unusual decays could be searched for also among the spontaneous fission events of superheavy nuclei [8].

The extreme clustering process of formation of two lead-like doubly magic fragments in collisions of actinide nuclei is also a very interesting subject for experimental study. Such measurements, in our opinion, are not too difficult. It is sufficient to detect two coincident lead-like ejectiles (or one lead-like and one calcium-like fragments) in $\mathrm{U}+\mathrm{U}$ collisions to conclude unambiguously about the ternary fission of the giant nuclear system.

\section{References}

1. C. Wagemans, Ternary Fission in The Nuclear Fission Process, edited by Cyriel Wagemans (CRC Press, Boca Raton, 1991), Chap. 12.

2. F. Gönnenwein et al., in Seminar on Fission, Pont d'Oye IV, Belgium, 1999, edited by Cyriel Wagemans et al., (World Scientific, Singapore, 1999) p. 59.

3. W.J. Swiatecki, Second UN Int. Conf. on the Peaceful Uses of Atomic Energy, Geneva, 1958, p. 651.

4. H. Diehl and W. Greiner, Nucl. Phys. A229, 29 (1974).

5. A.R. Degheidy, J.A. Maruhn, Z. Phys. A 290, 205 (1979).

6. H. Schulheis, R. Schulheis, Phys. Lett. B 49, 423 (1974).

7. N. Carjan, A.J. Sierk and J.R. Nix, Nucl. Phys. A452, 381 (1986).

8. Yuri Oganessian, J. Phys. G, 34, R165 (2007).

9. J. Maruhn and W. Greiner, Z. Phys. A 251, 431 (1972).

10. V.M. Strutinsky, Nucl. Phys. A 95, 420 (1967); Nucl. Phys. A 122, 1 (1968).

11. V.I. Zagrebaev, A.V. Karpov, Y. Aritomo, M. Naumenko, W. Greiner, Phys. Part. Nucl. 38, 469 (2007).

12. X. Wu, J. Maruhn and W. Greiner, J. Phys. G 10, 645 (1984).

13. D.V. Kamanin, Yu.V. Pyatkov, A.N. Tyukavkin and Yu.N. Kopatch, Int. J. Mod. Phys. E17, 2250 (2008).

14. V.I. Zagrebaev, Yu.Ts. Oganessian, M.G. Itkis and W. Greiner, Phys. Rev. C 73, 031602 (2006). 